\title{
A Botnet Detecting Infrastructure Using a Beneficial Botnet
}

\author{
Takashi Yamanoue \\ Fukuyama University \\ Sanzo, 1, Gakuen-cho \\ Fukuyama, Hiroshima, 729-0292 Japan \\ yamanoue@fukuyama-u.ac.jp
}

\begin{abstract}
A beneficial botnet, which tries to cope with technology of malicious botnets such as peer to peer (P2P) networking and Domain Generation Algorithm (DGA), is discussed. In order to cope with such botnets' technology, we are developing a beneficial botnet as an anti-bot measure, using our previous beneficial bot. The beneficial botnet is a group of beneficial bots. The peer to peer $(\mathrm{P} 2 \mathrm{P})$ communication of malicious botnet is hard to detect by a single Intrusion Detection System (IDS). Our beneficial botnet has the ability to detect P2P communication, using collaboration of our beneficial bots. The beneficial bot could detect communication of the pseudo botnet which mimics malicious botnet communication. Our beneficial botnet may also detect communication using DGA. Furthermore, our beneficial botnet has ability to cope with new technology of new botnets, because our beneficial botnet has the ability to evolve, as same as malicious botnets.
\end{abstract}

\section{CCS CONCEPTS}

- Security and privacy $\rightarrow$ Intrusion/anomaly detection and malware mitigation $\rightarrow$ Intrusion detection systems

\section{KEYWORDS}

Bot, Security, Collaboration

\section{ACM Reference format:}

T. Yamanoue. 2018. A Botnet Detecting Infrastructure Using a Beneficial Botnet. In Proceedings of ACM SIGUCCS, Orland, Florida USA, October 7-10, 2018, ACM, NY, NY, USA., 8 pages. https://doi.org/10.1145/3235715.3235728

\section{Introduction}

Many network managers and security managers are having trouble with malicious botnets. [7] A botnet spreads many spam mails to many people from many places, sends many packets to a

\footnotetext{
Permission to make digital or hard copies of all or part of this work for personal or classroom use is granted without fee provided that copies are not made or distributed for profit or commercial advantage and that copies bear this notice and the full citation on the first page. Copyrights for components of this work owned by others than ACM must be honored. Abstracting with credit is permitted. To copy otherwise, or republish, to post on servers or to redistribute to lists, requires prior specific permission and/or a fee. Request permissions from Permissions@acm.org.

SIGUCCS '18, October 7-10, 2018, Orlando, FL, USA

(C) 2018 Association for Computing Machinery.

ACM ISBN 978-1-4503-5582-7/18/10 ..\$15.00

https://doi.org/10.1145/3235715.3235728
}

server in a short time to perform DDoS attacks, using its massively parallel feature. Bots of the botnet may steal bank account information of users of zombie computers. The botnet is also persistent. Even if security managers found out some of bots of the botnet, the botnet continues to do malicious things. Malicious botnets are evolving every day in order to survive the managers' anti-bot measures. Latest botnets adopt peer to peer (P2P) networking, Domain Generation Algorithm (DGA), and other technologies for surviving the anti-bot measures. In order to cope with such botnets' technology, we are developing a beneficial botnet as an anti-bot measure, using our previous beneficial bot [11] [12].

Our beneficial botnet is a group of beneficial bots which are agent bots and an analyzing bot. An agent bot is located between a LAN and its network address translator (NAT)/ router. The agent bot collects and controls communication of hosts in the LAN. The analyzing bot collects communication data from agent bots and analyzes the data. The analyzing bot can execute $\mathrm{R}$ programs of the R statistic computing system in order to analyze the data. The $\mathrm{P} 2 \mathrm{P}$ communication of malicious botnet is hard to detect by a single Intrusion Detection System (IDS) at the entrance of organizational network, because there may be a small amount of traffic of $\mathrm{P} 2 \mathrm{P}$ communication between $\mathrm{P} 2 \mathrm{P}$ nodes at the inside of the organizational network and the outside. Our beneficial botnet has the ability to detect $\mathrm{P} 2 \mathrm{P}$ communication, using collaboration of our beneficial bots.

We have made a pseudo botnet, pseudo Gameover ZeuS, which performs $\mathrm{P} 2 \mathrm{P}$ communication between some nodes of it without doing malicious things, in order to evaluate our beneficial botnet.

Our beneficial botnet could detect P2P communication of the pseudo Gameover ZeuS in the experimental botnet detecting infrastructure which is constructed using our beneficial botnet. Our beneficial botnet may also detect communication using DGA. Furthermore, our beneficial botnet has ability to cope with new technology of new botnets, because our beneficial botnet has the ability to evolving, as same as malicious botnets.

\section{Beneficial Botnet}

It is common to use an IDS or intrusion prevention system (IPS) at the entrance of an organizational network now. An IDS or IPS at the entrance of the organizational network is effective for a malicious botnet with centralized communication and control (C2) server because every communication between the $\mathrm{C} 2$ server and all bots can be detected by the IDS or the IPS. However, it is 
hard to detect the P2P communication between bots of the P2P malicious botnet in the organizational network by the IDS or IPS at the entrance of the network.

Figure 1 shows the outline of the beneficial botnet. The beneficial botnet consists of agent bots and an analyzing bot. An agent bot of our beneficial botnet is placed between a sub-LAN and its NAT or router, and the agent bot collects communication data between the sub-LAN and outside of the sub-LAN. The analyzing bot analyzes the collected communication data from all of agent bots and detects bots in the sub-LANs. Every agent bot or the analyzing bot is controlled by the script in the wiki page of a wiki site in the Internet.

Gameover ZeuS [1] is a famous botnet. It was disrupted in 2014 by the international collaborative investigating activity. However, the losses attributable to Gameover ZeuS were estimated over one hundred million dollars according to the FBI announcement [3]. "The second centralized version of Zeus mutated into a peer-topeer (P2P) variant, known as P2P Zeus or Gameover. Since P2P Zeus does not rely on centralized command and control server (C2), it is immune to traditional countermeasures against Zeus", according to Andriesse and Bos [1]. The P2P feature of botnets makes botnets difficult to detect and difficult to disrupt. So, the Gameover ZeuS could steal large amounts of money and it took big effort, such as international collaboration, to disrupt it.

The Gameover ZeuS has been disrupted but there can be similar P2P botnet as the Gameover ZeuS in organizational networks. We have designed our beneficial botnet to cope with malicious botnet such as the Gameover ZeuS.

\subsection{Bot of the Beneficial Bot-Net}

A bot of our beneficial bots is a script interpreter, the script of which is in the wiki page of a wiki software. We use PukiWiki as the wiki software. [13] [14] [15]

Figure 2 shows the behavior of our bot. The bot repeats the following:

1) Wait for the designated time which will be replaced by the "set readInterval" command in the script.

2) Read the script, which consists of commands and a program, from the wiki page from the specific Wiki site which is assigned to the bot.

3) Execute these commands and the program. The program can read other wiki pages and Web pages. The program also can read data from its attachment and can send command to the attachment.

4) Write results of the execution to the send buffer. Data in the send buffer are written back to the wiki page which contains the commands and the program.

The series of commands on the wiki page is read and interpreted when the page has it. If a program is embedded in the series of commands, then the program is transferred to the language processor of the bot. The program is translated into its internal representation. The internal representation of the program is evaluated by the interpreter.

The attachments in the Figure 2can be sensors, actuators and other functions. In the case of an agent bot, the attachment of the agent bot is the traffic controller which is shown later.

Figure 3 shows an example of the wiki page which includes the script for our bot and the results of execution. In this example, lines which start with "command:" are the commands.

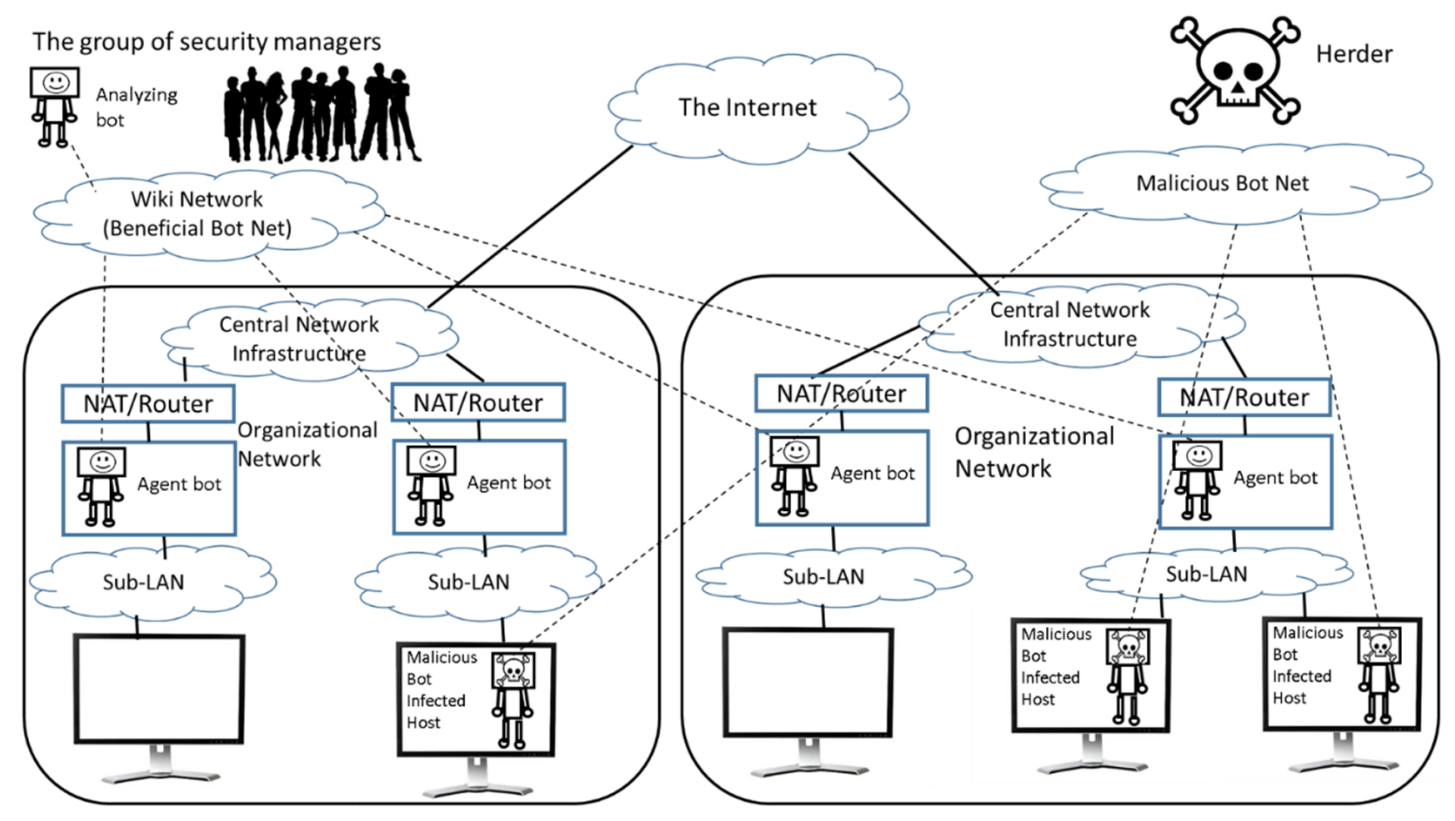

Figure 1 Outline of the Beneficial Bot-net 


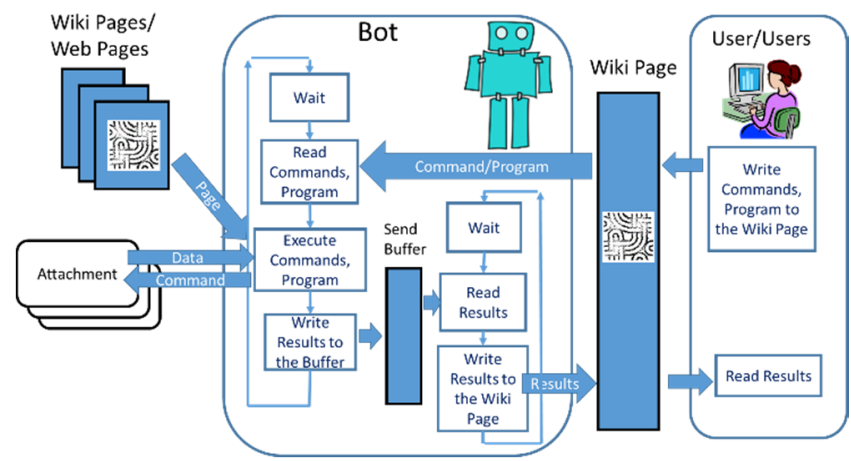

Figure 2 Behavior of a bot

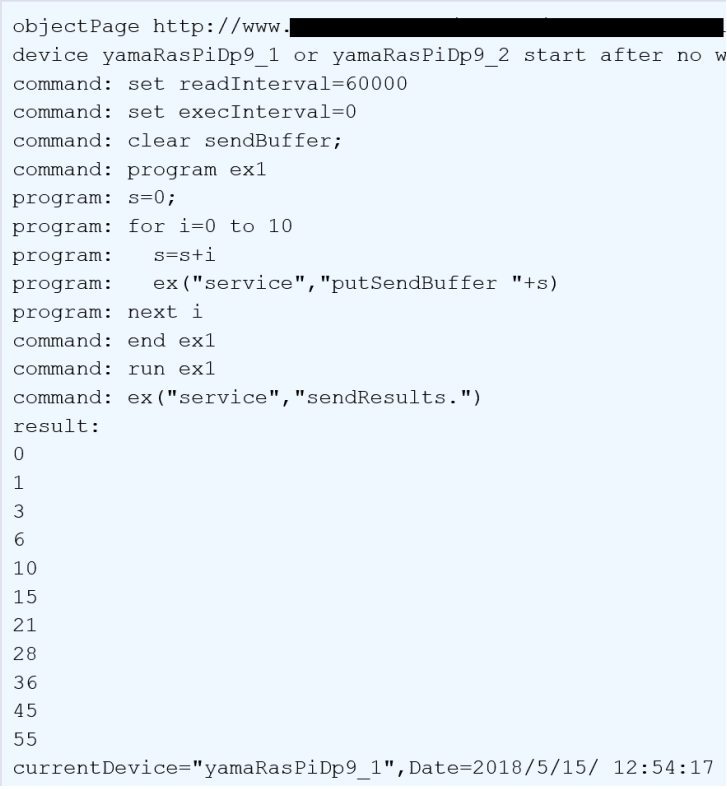

Figure 3 Example of the Script for a beneficial bot

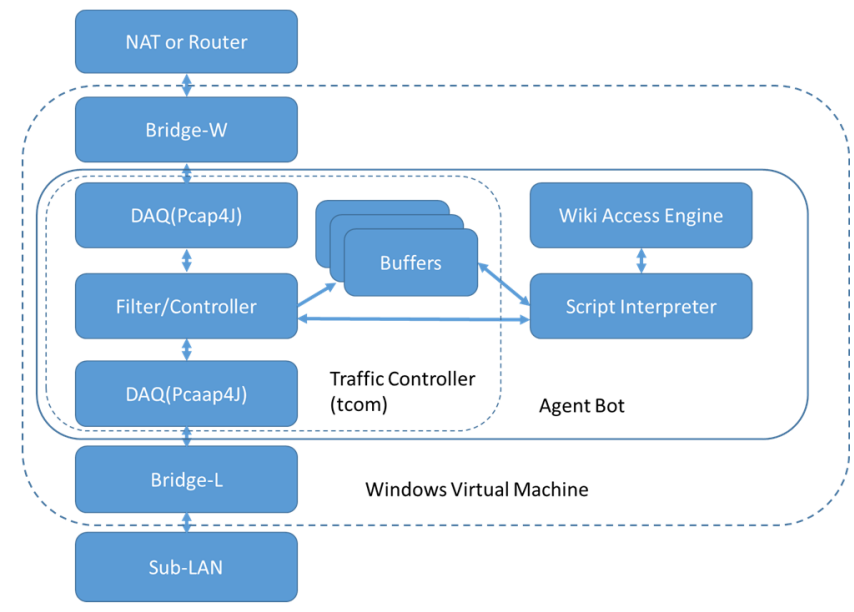

Figure 4 Structure of the Agent Bot
The line of "command: set readInterval=600000" shows that the mobile terminal reads the page of the given URL every one minutes after that. The right hand side of the equation shows the interval time in milliseconds. Lines which start with "program:" are lines of the program. A program is enclosed by lines of "command: program ex" and "command: end ex". In this example, the program is named "ex" by these lines. The "command: run ex" shows that the program "ex" is translated into its internal representation and executed after that when this command line is interpreted.

A wiki page for the bot also can contain the "set pageName" command and the "include" command. When the "set pageName" command is interpreted in the bot, the bot will use the wiki page which is designated by the set pageName command, as the main wiki page, next time.

The page name of the designated wiki page can include the current time or the current date. For example, when the "set pageName $=$ "pir-1-<hour $>$ "' was interpreted at the time of eight o'clock, the bot use the page of "pir-1-8", in the same wiki server of the current wiki page, as the main wiki page, next time.

When the "include" command is interpreted in the bot, the bot inserts the wiki page, which is designated by the include command, into the place at the include command of the original wiki page. This command is useful when there are identical commands or program in many of wiki pages. We call the included wiki page as a class wiki page just like a class of an object oriented programming language. A class wiki page can also include other wiki pages just like super classes of an object oriented programming language.

We call the wiki page, which contains the commands, the program, and data which are written back from the bot, as the main wiki page. Commands and the program of the main wiki page can be modified to change the behavior of the bot without stopping the bot. We also call the main wiki page as an object wiki page just like an object of an object oriented programming language.

The bot can be connected to a LAN which is protected by a router with the NAT or NAPT function. The manager of the bot can control the bot from the outside of the LAN.

\subsection{Agent Bot}

An agent bot is a bot of the beneficial botnet, with the traffic controller (tcon). The traffic controller has two network interfaces, two data acquisition library (DAQ), packet filter/controller, and information buffers. We use Pcap4J [6] for the DAQ. The script interpreter of the bot operates the tcon. The bot collects communication data between its two interfaces and writes the data to the wiki page, according to the script on the wiki page. It can also control the communication between two interfaces. Connecting to one network interface to the NAT or router, and another network interface to the sub-LAN, allows the bot to collect and control every communication between the Sub-LAN and outside of the Sub-LAN. Figure 4 shows the structure of the agent bot. 
An agent bot has following buffers. Communication data are classified and stored to these buffers according to the kind of data.

\section{- Packet-history}

This buffer contains packet information that is transferred between two interfaces. This buffer has sub buffers for packets with the same pair of the source IP address and the destination IP address. Packet information is stored in these sub buffers separated and sorted by the pair. Also stored are the sha1 hash value of the packet payload, time of its transfer, and packet information. The sha1 value is used to detect P2P communication between two hosts in separated sub-LAN. When a sub buffer is overflowed, old information is deleted.

\section{- MAC-list}

This buffer contains all of MAC addresses in the all of frames in this sub-LAN. Every IP address which is resolved from the MAC address is also stored associated to the MAC address. This list can be used to know which hosts are connected to this sub-LAN and its IP addresses in this LAN. Default gateway can be identified if the network interface of its MAC address has many IP addresses than others.

\section{- Domain-list}

This buffer contains results of DNS queries in this subLAN with the time of its query. This list can be used to know which host in this sub-LAN communicated with which host outside of this LAN. This list also can be used to detect the usage of Domain Generation Algorithm (DGA).

\section{- DHCP-list}

This buffer contains results of DHCP communication with the time of the communication. This list can be used to detect the DHCP spoofing or to detect un-authorized DHCP server.

- Arp-list

This buffer contains results of Arp communication with the time of the communication. This list can be used to detect the Arp spoofing.

There are commands to get information from and manipulate these buffers, as well as commands to control the communication between two network interfaces. These commands can be used to deny the activity of malicious bots.

\subsection{Analyzing Bot}

The analyzing bot gathers information of each agent bot and analyzes them. The language processor of all beneficial bots is equipped with the comma separated value (CSV) parser and table manipulate/calculate functions. The analyzing bot uses the $\mathrm{R}$ statistical computing system [4] to analyze the data. R expressions can be used in the script for the analyzing bot. Value transformation functions between the original language processor of bots and R statistical computing system are also available.

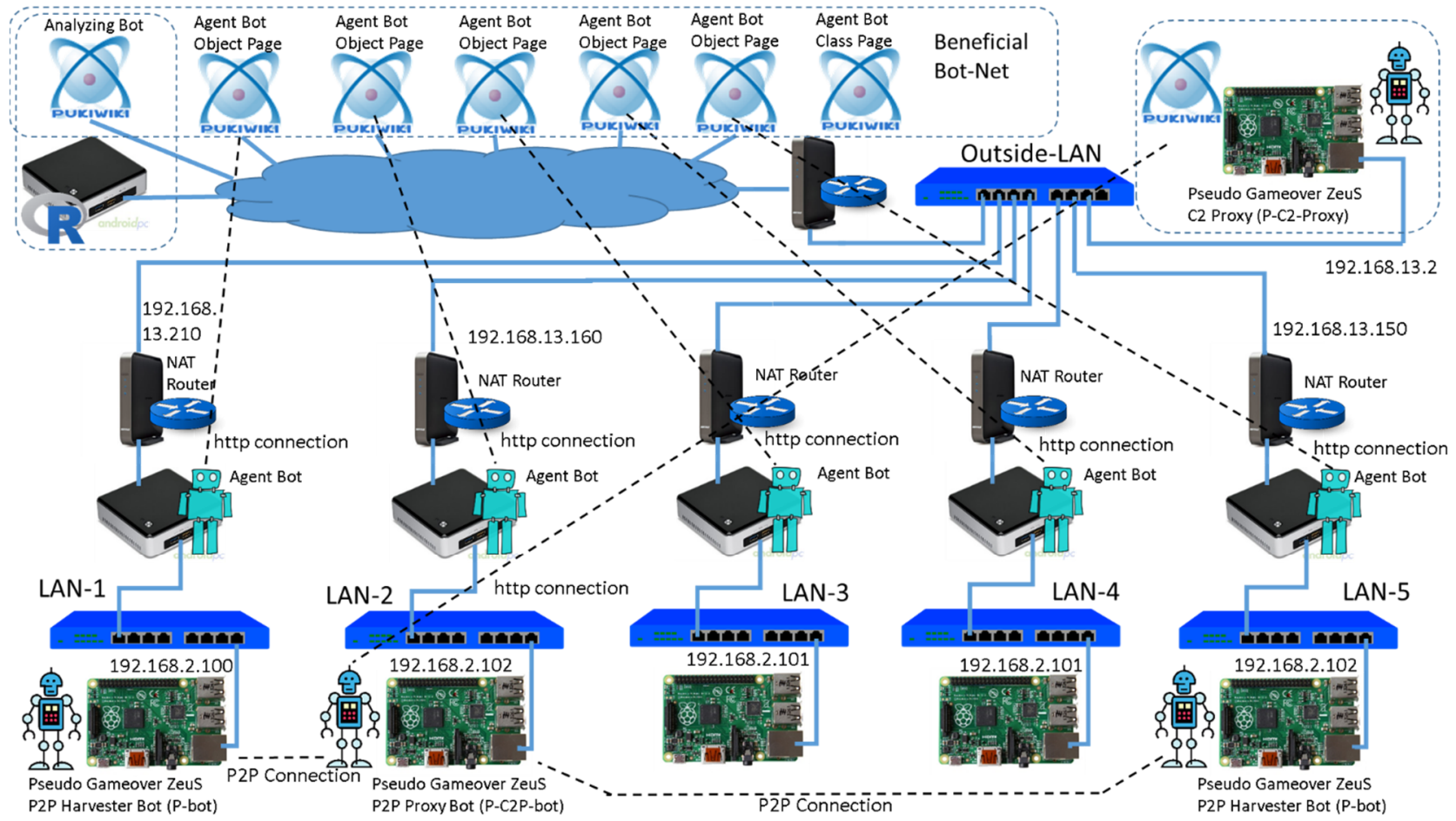

Figure 5 Experiment using the beneficial botnet and pseudo Gameover ZeuS. 


\section{Experiment}

We have implemented an experimental bot detecting infrastructure using our beneficial botnet.

In order to evaluate the usefulness of the infrastructure, we also have implemented pseudo Gameover ZeuS which performs P2P communication between bots of them without performing malicious things. We have conducted an experiment using these implementations, in order to know whether the beneficial botnet can detect P2P communication or not. Figure 5 shows the network for the experiment.

We have used five agent bots and one analyzing bot as the beneficial botnet. Each agent bot and its main wiki page is connected by the http connection. Analyzing bot and its main wiki page is also connected by the http connection. All of main wiki pages for agent bots include the agent bot class page. IP addresses above NAT routers show their IP addresses at Outside-LAN. The hardware of each bot of the beneficial botnet was an Intel NUC. VMWwas also used to copy the one agent bot with its running environment to other machines.

The pseudo Gameover ZeuS consists of P2P Harvester bots $(P-$ bot), P2P Proxy bots (P-C2P-bot) and a C2 proxy (P-C2-Proxy). We have used two $\mathrm{P} 2 \mathrm{P}$ harvester bots, one $\mathrm{P} 2 \mathrm{P}$ proxy bot and one $\mathrm{C} 2$ proxy. The P-C2-Proxy has a simplified STUN server for initiating $\mathrm{P} 2 \mathrm{P}$ communication between two harvester bots and the proxy bot. The P-C2P-bot reads the command in the web page of the $\mathrm{P}-\mathrm{C} 2$ Proxy periodically and spread the command to P-bots using the P2P network. UDP is used for the P2P communication. We have placed one P-bot in the LAN-1, another P-bot in the LAN-5, the PC2P-bot in the LAN-2, and the P-C2-Proxy in the Outside-LAN. The IP addresses of them are 192.168.2.100, 192.168.2.102, 192.168.2.102, and 192.168.13.2, respectively. We have implemented these bots using a Raspberry Pi.

\subsection{Script and results of Agent Bots}

We have directed every agent bot to get information about possible packets, which may be packets of $\mathrm{P} 2 \mathrm{P}$ communication of pseudo Gameover ZeuS, by the script of the wiki page. A possible packet should have the following features.

- It must be UDP packet because UDP is used for P2P communication of Gameover ZeuS.

- $\quad$ It must not use the port 123 because the port number is used for NTP and a pair of ephemeral ports is used for P2P communication.

- $\quad$ It must not use the port 53 because the port number is used for DNS query and a pair of ephemeral ports is used for P2P communication.

- It must be sent and received repeatedly during the action of the Gameover ZeuS.

Figure 6 shows the script of the class page which directs all agent bots to get information of the above. In Figure 6, the line of "program: ex("tcon", "set repeating number $=20$.")" directs the bot to gets information of at most 20 latest packet information of each sub buffers, when the "get repeating" command is executed. The "tcon" shows the traffic controller of the agent bot. The information in the variable output10 is represented by lines of packet information in text.

The line of "program: output10=ex("tcon","get repeating unicast over 60000.")" shows getting unicast packet information, which is sent/received repeatedly more than one minutes (60000 msec.), of all sub buffers, and store the information to the variable output10. The following is an example of one line of the output lines.

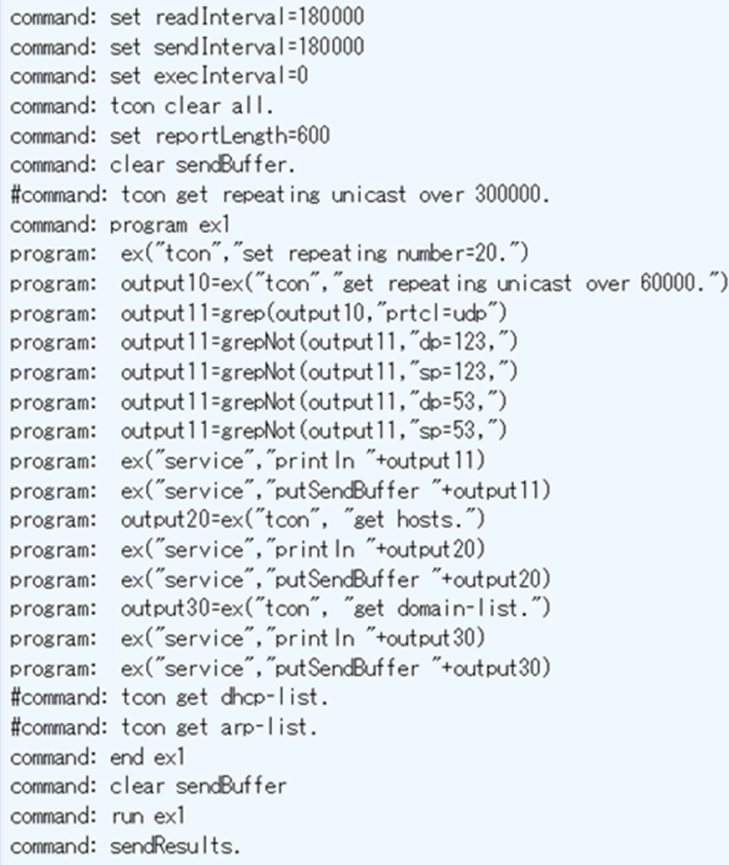

Figure 6 Script of the Class page to direct all of agent bots

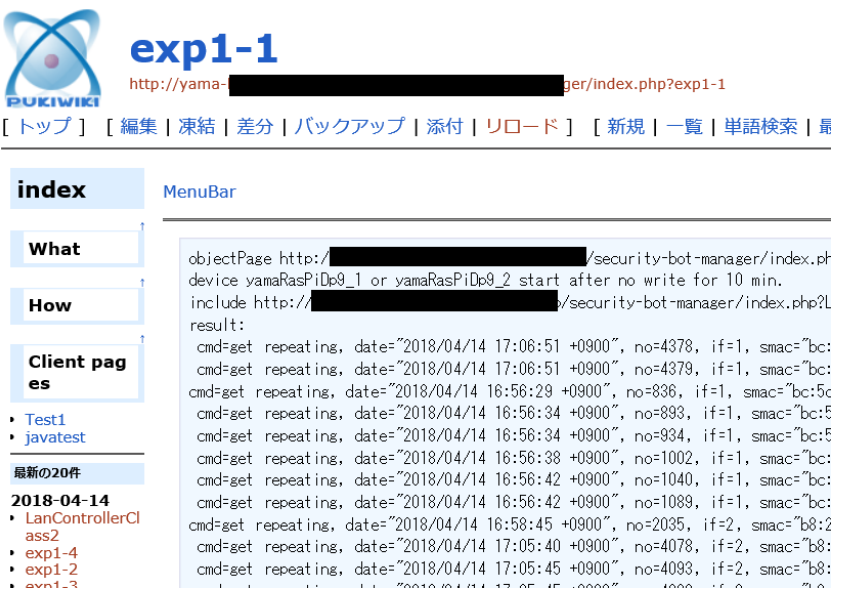

Figure 7 A part of the object page for an agent bot

$c m d=$ get repeating, date $=" 2018 / 04 / 14$ 17:03:19 $+0900 "$, no=3299, if $=1, \quad$ smac $=" b c: 5 c: 4 c: 5 d: 1 c: c d ", \quad d m a c=" b 8: 27: e b: c b: d 6: 38 "$, 
prtcl=udp, sip="192.168.13.160", dip="192.168.2.100", $s p=34724$, $d p=33331$, sha1payload="9dac7a7beb944a7193847a3d0fbcc370d13a5838", payloadLength $=46, \quad$ payload=broadcast $i d=3394824 \quad t t l=3$ cmd $=$ "message test".....

The "cmd=get repeating" shows that this information is outputted by the command "get repeating". The "date="2018/04/14 17:03:19 +0900" shows the date and time of the packet transferred. The "no=3299" shows the serial number of the packet in the traffic

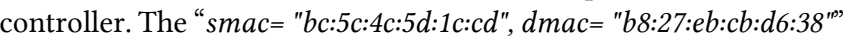
shows the source and destination MAC addresses of this packet. The "prtcl=udp" shows the protocol of this packet. The "sip="192.168.13.160", dip="192.168.2.100"s" shows the source and destination IP addresses of this packet. The " $s p=34724, d p=33331$ " shows the source and destination port number of this packet. The "sha1payload = "9dac7a7beb944a7193847a3dofbcc 370d13a5838" shows the sha1 hash value of the payload of this packet. The "payloadLength $=46$ " shows the payload length (the byte number) of this packet. The "payload=broadcast id=3394824 $t t l=3$ cmd $="$ message test"....." shows the head of the payload of this packet.

The line of "program: output11=grep(output10,"prtcl=udp")" shows extracting the UDP packet from the information in the variable output 10 and storing the information to the variable output11. The packet information of the above example is extracted by this command because the information includes the "prtcl=udp". The line of "program: output11 = grepNot ( output11," $d p=123$,")" shows extracting information of the packet, the destination port of which is not 123, and storing the information to the variable output11. The packet information of the above example is extracted because the information does not include " $d p=123$, . The line of "program: output11 = grepNot ( output11,"sp=123, “)" shows extracting information of the packet, the source port of which is not 123 (NTP), and storing the information to the variable output11. The line of "program: output11 = grepNot ( output11,"dp=53,")" shows extracting information of the packet, the destination port of which is not 53 (DNS), and storing the information to the variable output11. The line of "program: output11 = grepNot ( output11,"sp=53,")" shows extracting information of the packet, the source port of which is not 53, and storing the information to the variable output11. After all, the packet information of the above example is extracted by these commands in the program.

The script of the Figure 6 also output the host list and domain (DNS query) list in the sub LAN. The host list can be used to know how many and which kind of hosts are connected to the sub LAN. Routers also can be detected because routers usually have many IP addresses with one MAC address. The domain list can be used for detecting the DGA because a malware with DGA usually conducts a large number of DNS queries of strange FQDN periodically. If there are a large number of strange DNS queries, the domain list becomes large with strange DNS queries from one host. Many of the DNS queries should be failed.
The agent bot also can use commands of "get dhcp-list" and "get arp-list". They can be used for detecting DHCP spoofing and ARP spoofing.

Figure 7 shows a part of the object page for an agent bot. This page includes the line which includes the class page of Figure 6. Network information of the sub LAN, which is captured by the script of Figure 6, is shown after the line of "result:".

\subsection{Script and results of Analyzing Bot}

Figure 8 shows the script of the analyzing bot. This script reads all of the output information of agent bots on their object wiki pages, analyzes the information, and outputs the information which shows a possible P2P packet from one sub LAN to another if there is.

The manager of the beneficial botnet also can know the MAC addresses and IP addresses of hosts which can be infected by malwares.

The part (a) of the Figure 8 is the head part of the script. There is the program for $\mathrm{R}$ statistical computation system between the lines of "command: $\mathrm{R}$ ex1" and "command: end ex1". The $\mathrm{R}$ program in this part includes the definition of the function "p2pPacketList". The ";;" at the end of lines of the function shows that the definition of the function continues after this line. This function is used for extracting packets which seems transferred from one LAN from another.

The part (b) of the Figure 8 shows the head of the program ex2. The program ex 2 extracts required information for the analyze from all of the object wiki page of the agent bots, passes the information to the R function of the part (a), and output the results on the object wiki page of the analyzing bot. This part includes definitions of arrays which are used in this program and the URLs of object wiki pages of all agent bots.

The part (c) of the Figure 9 shows the middle part of the program ex2. This part reads the information of each object page of agent bots and prepare the data for analyzing by the $\mathrm{R}$ function.

The part (d) of the Figure 9 shows the tail part of the program ex2. This part receives the results of analyzing the data from the $\mathrm{R}$ function, transform the results to the text for writing back to the object wiki page, and writes back to the page.

The results in the object wiki page consists of lines. Each line of them shows that the possible packet, which must be used for P2P communication, was transferred between two sub LANs.

When the experiment was conducted, we had 70 lines which show the possible communication between two sub LANs. The following is a line in the result part of the object wiki page.

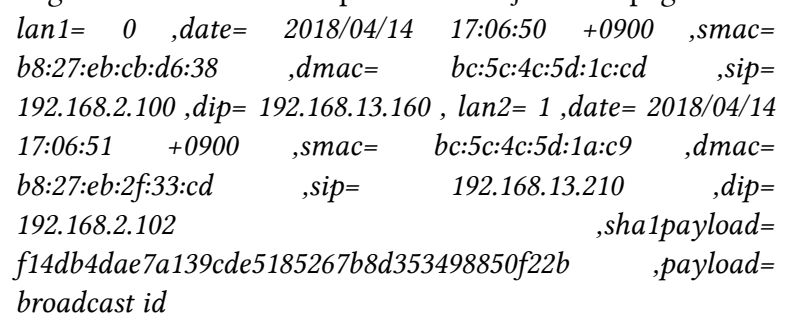

The "lan $1=0$ " and the "lan $2=1$ " shows that the packet seems transferred between LAN-1 and LAN-2. The "date= 2018/04/14 
17:06:50 +0900" and the "date $=2018 / 04 / 1417: 06: 51+0900$ " shows the time of the transferring. The "smac $=b 8: 27: e b: c b: d 6: 38, d m a c=$ $b c: 5 c: 4 c: 5 d: 1 c: c d$ " shows the source MAC address and the destination MAC address of the packet in the LAN-1. The "smac= $b c: 5 c: 4 c: 5 d: 1 a: c 9, d m a c=b 8: 27: e b: 2 f: 33: c d$ " shows the source MAC address and the destination MAC address in the LAN-2. The "sip= 192.168.2.100, dip=192.168. 13.160" shows the source IP address and the destination IP address of the packet in the LAN-1, and the "sip= 192.168.13. $210, d i p=192.168 .2 .102 "$ shows the source IP address and the destination IP address of the packet in the sub LAN-2.

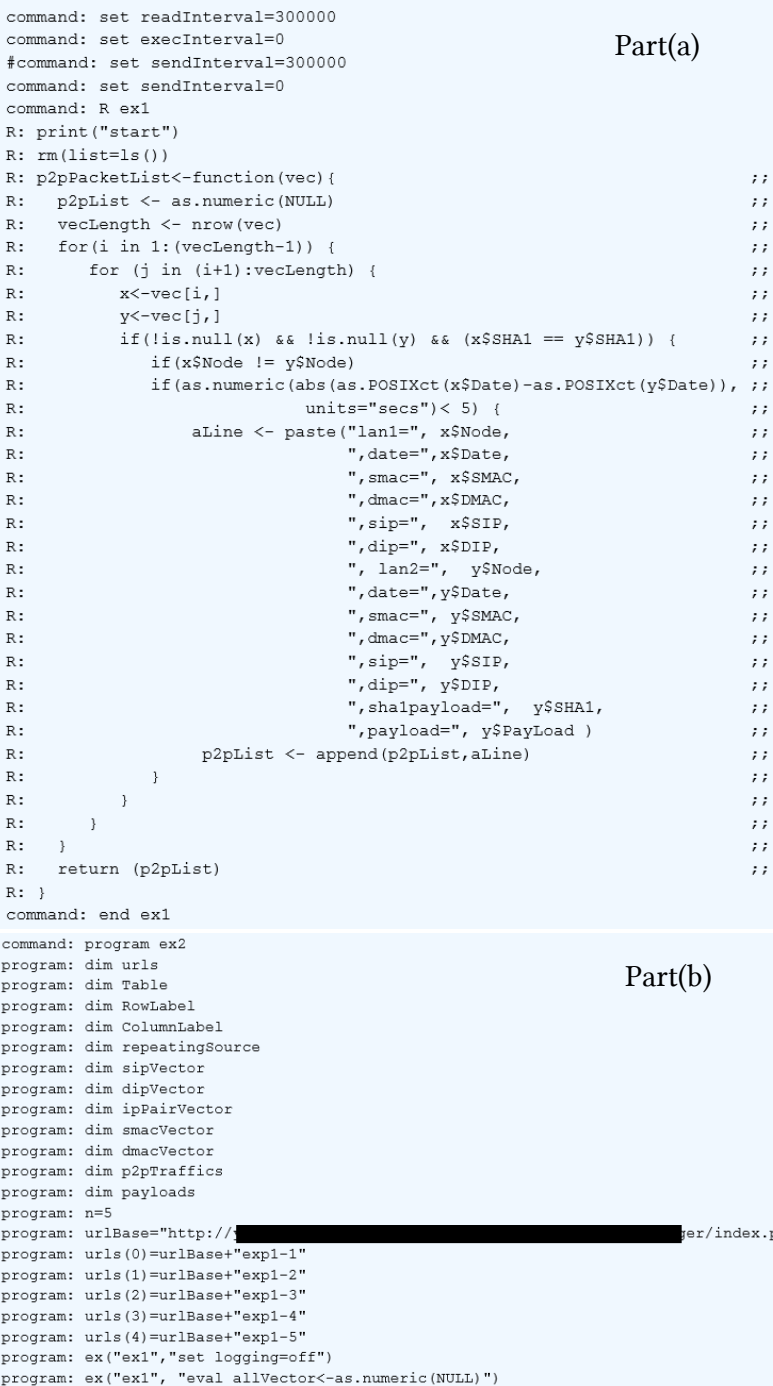

Figure 8 Script of the Analyzing Bot, Part (a) and (b)

We can presume the packet flow, which is shown by the dotted arrows in the Figure 10, by this information. The "sha1payload= ..., payload= broadcast id" shows the sha1 of the payload of the transferred packet and a part of the payload. There was also the following line which indicates the packet flow between LAN-2 and LAN-5 in the object wiki page.

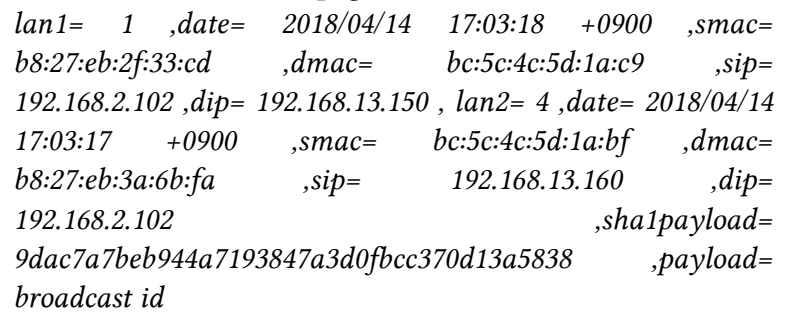

There was no line which indicates the P2P communication from or to LAN-3 and LAN-4.

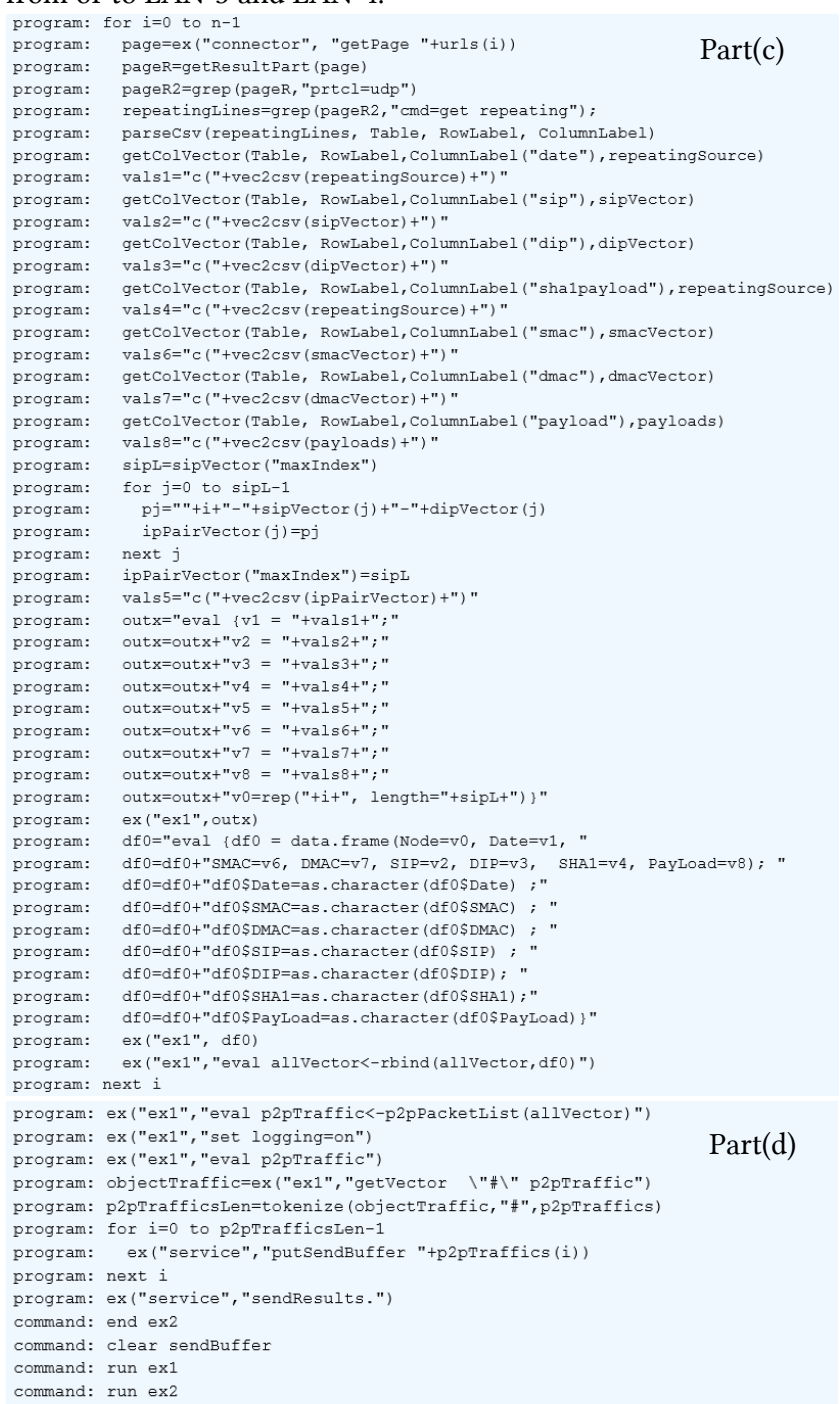

Figure 9 Script of the Analyzing Bot, Part (c) and (d) 


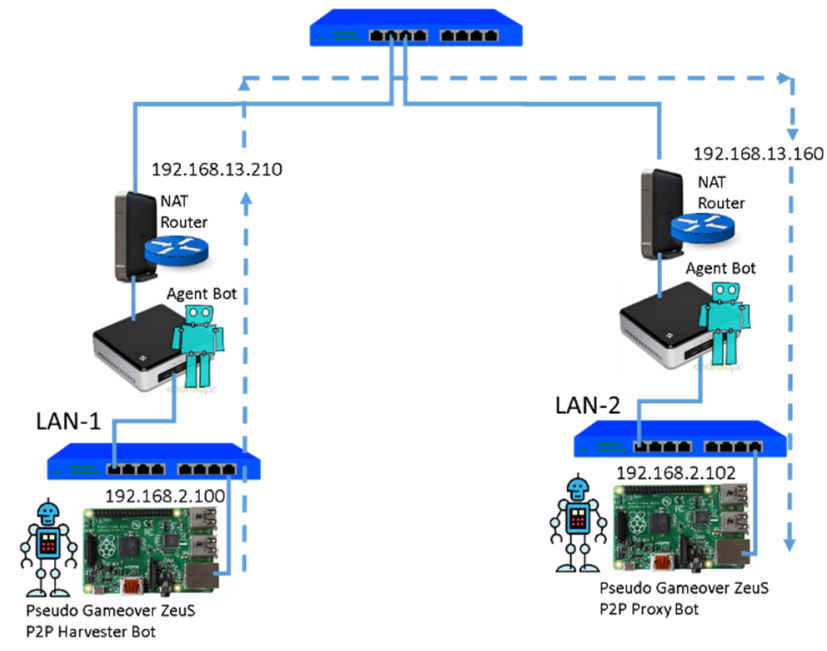

Figure 10 Possible packet flow of the line of "lan $1=0, \ldots$ $, \operatorname{sip}=192.168 .2 .100, \operatorname{dip}=192.168 .13 .160, \operatorname{lan} 2=1, \ldots, \operatorname{sip}=$ 192.168.13.210 $, \operatorname{dip}=192.168 .2 .102, \ldots ”$

\section{Related Work}

\subsection{AAFID}

Autonomous Agents for Intrusion Detection (AAFID) [9] are agents for a distributed intrusion detection system as same as our beneficial botnet. This system consists of agents, transceivers and monitors. An agent of the AAIF is similar to our agent bot in that both of them are controlled by commands and collect traffic data. An agent of the AAIF is installed in a client host while our agent bot is placed between LAN and its router or NAT router. The manager of our beneficial botnet does not need to install our agent bot to each client host. A monitor and a transceiver of the AAIF is similar to our analyzing bot in that both of them are collecting data from agents, transceivers, other monitors or agent bots, and analyzing the data. A monitor or a transceiver of the AAIF is not controlled by the script in a wiki page while our agent bots and the analyzing bot is controlled by the script in a wiki page. Communication mechanism is not specified in the AAFID architecture while our beneficial botnet uses wiki API.

\subsection{Man-in-the-Middle attack}

The action of an agent bot of our beneficial bot can be seen as the man-in-the-middle attack. [2] Many communications in the subLAN can be controlled by the agent bot. We have to be careful so that the agent bot does not to go to the dark side.

\subsection{KASEYA and UNIFAS}

The PC management system of KASEYA [5] and the wi-fi access point management system of Furuno Systems (UNIFAS) [10] consist of agent programs at the devices, such as PCs or wi-fi access points, and a web site to manage them, as in our beneficial botnet. Their devices can also communicate with the web site over a NAT. However, they use a specialized web server, whereas our beneficial botnet uses a web site with common wiki software.

\section{CONCLUSION}

We showed that P2P communication by malware can be detected by our beneficial botnet. The possible use of DGA algorithm can be also detected. Our beneficial botnet consists of bots which are script interpreters and wiki pages for storing the scripts. So, our beneficial botnet has the ability to cope with new technology of new botnets by rewriting the scripts.

Currently, traffic speed between LAN and WAN over an agent bot is very slow. We have to improve the speed for real use of our beneficial botnet. We also have to improve the security of our beneficial botnet.

\section{ACKNOWLEDGMENTS}

A part of this research was supported by JSPS KAKENHI Grant Number JP16K00197. We thank the author of Pcap4J! and the community of R. We also thank students who helped us to conduct the experiment in this paper.

\section{REFERENCES}

[1] Andriesse, D. and Bos, H. 2014 An Analysis of the Zeus Peer-to-Peer Protocol, Technical Report IR-CS-74, rev. April 10, 2014.

[2] Conti, M., Dragoni, N. and Lesyk, V. 2016 A Survey of Man In The Middle Attacks, IEEE Communications Surveys \& Tutorials, Vol. 18, Issue 3, 2016. IEEE, 2027-2051. DOI=10.1109/COMST.2016.2548426

[3] FBI. 2014. GameOver Zeus Botnet Disrupted, https://www.fbi.gov/news/stories/gameover-zeus-botnet-disrupted, June 2, 2014

[4] Ihaka, R., and R. Gentleman. 1996. R: a language for data analysis and graphics. J. Comp. Graph. Stat. 5:299-314. Available via http://www.Rproject.org.

[5] KASEYA, http://www.kaseya.com/

[6] Pcap4J, https://www.pcap4j.org

[7] Puri, R. 2003. Bots \& Botnet: An Overview," SANS InfoSec Reading Room, http://www.sans.org/rr/whitepapers/malicious/, Dec., 2003.

[9] Spafford, E. H. and Zamboni, D. 2000 Intrusion detection using autonomous agents, Computer Networks vol.34, pp.547-570, Elsevier, 2000 .

[10] UNIFAS, http://www.furunosystems.co.jp/product/detail/unifas.html

[11] Yamanoue, T., Oda, K., Shimozono. K. 2012. Capturing Malicious Bots using a Beneficial Bot and Wiki, In Proceedings of the 40th annual ACM SIGUCCS conference on User services (Memphis, Tennessee, USA. 15-19 Oct. 2012). ACM, New York, NY, 91-96. DOI=https://doi.org/10.1145/2382456.2382477

[12] Takashi Yamanoue, Kentaro Oda, Koichi Shimozono. A Malicious Bot Capturing System using a Beneficial Bot and Wiki, Journal of Information Processing(JIP), vol.21, No.2, pp.237-245(2013).

[13] Yamanoue, T., Oda, K., Shimozono. K. 2013. An Inter-Wiki page Data Processor for a M2M System, In Proceedings of the 4th International Conference on E-Service and Knowledge Management (ESKM 2013), Advanced Applied Informatics (IIAIAAI), 2013 IIAI International Conference on.(Matsue, Shimane, Japan, 31 Aug- 4 Sep. 2013) IEEE, Los Alamitos, CA. 45-50. DOI= https://doi.org/10.1109/IIAI-AAI.2013.48

[14] Yamanoue, T., Oda, K., Shimozono. K. 2014. Experimental Implementation of a M2M System Controlled by a Wiki Network, In Applied Computing and Information Technology, Studies in Computational Intelligence, Springer, Vol.553, 121-136.

[15] Yamanoue, T., .2017. Monitoring Servers, With a Little Help from my Bots, In Proceedings of the 45th annual ACM SIGUCCS conference on User services (Seattle, Washington, USA. 01-04 Oct. 2017). ACM, New York, NY, 173-180. DOI=https://doi.org/10.1145/3123458.3123461 